\title{
Application of Motivational Interviews in Primary Care for Obese Children
}

\author{
Yuanyuan Fang ${ }^{1}$, Jie Yao ${ }^{1, *}$, Fang Liu ${ }^{1}$, Xiaowei Li $^{1}$, Bo Dong ${ }^{2}$ \\ ${ }^{1}$ Department of Nursing, Shaanxi University of Traditional Chinese Medicine, Xianyang City, Shaanxi Province 712046, China. \\ ${ }^{2}$ Department of Orthopedics, Affiliated Hospital of Shaanxi University of Traditional Chinese Medicine, Xianyang 712000, Shaanxi \\ Province, China.
}

How to cite this paper: Yuanyuan Fang, Jie Yao, Fang Liu, Xiaowei Li, Bo Dong. (2021) Application of Motivational Interviews in Primary Care for Obese Children. International Journal of Clinical and Experimental Medicine Research, 5(1), 6-10.

DOI: 10.26855/ijcemr.2021.01.002

Received: November 30, 2020

Accepted: December 24, 2020

Published: December 31, 2020

*Corresponding author: Jie Yao, Department of Nursing, Shaanxi University of Traditional Chinese Medicine, Xianyang City, Shaanxi Province 712046, China.

Email: 344246303@qq.com

\begin{abstract}
This paper reviews the current situation and self-management level of obese children in China, and considers that motivational interviewing is a promising therapy in weight control. It also reviews the specific application of motivational interviewing in primary care of obese children, including the applied population, strategies and effects Evaluations suggest that motivational interviews help obese children evoke inner motivation, and then spontaneously change their eating habits and lifestyle, maintain long-term motivation, and finally reach their target weight. The analysis summarizes the current problems in the implementation of motivated interviews, and proposes that the motivated interviewers before the intervention lack standardized training, the implementation plan has no unified standards, and no mention of remuneration and other issues. It is recommended to improve the self-management level of children, increase the frequency of motivational interviews and give appropriate material rewards, pay attention to multi-disciplinary cross-cooperation and the application of artificial intelligence, accumulate experience in continuous implementation, and further Provide reference for promotion and application.
\end{abstract}

\section{Keywords}

Motivational Interviews, Obese Children, Primary Care, Review

\section{Introduction}

According to the statistics of the World Health Organization in 2016, more than 41 million children under the age of 5 have overweight or obesity [1]. At present, the overweight and obesity rate of children in developing countries is lower than that in developed countries, but its growth rate is no less than that of developed countries; if effective preventive measures are not taken in time, they will catch up with or even surpass developed countries in a short time [2]. In 2014, the overweight rate of school-age children over 7 years old in China also increased from $2.1 \%$ to $12.2 \%$, the obesity rate increased from $0.5 \%$ to $7.3 \%$, and the number of overweight and obese children increased from 6.15 million to 34.96 million [3]. Childhood obesity has become a world health problem, and is also the inducing factor of many chronic diseases. Childhood is a key period in the life cycle related to the development of physical and mental health. The nutritional status of this period plays an extremely important role in the future physical and mental health. Obesity not only affects children's normal growth and development and mental health, but also has potential harm to their adult life quality [4]. Motivational interviewing (MI), a patient-centered and goal-oriented communication model [5], aims to help people without behavior change motivation to change un- 
healthy behavior patterns, and has been widely used in the field of chronic disease management and general practice $[6,7]$. As a kind of disease-related risk factors, primary health care is the front-line setting for prevention of disease [8], which is complementary to MI. Therefore, the application of MI in primary health care for childhood obesity is a very good intervention measure.

\section{The Connotation of MI}

\subsection{Concept of MI}

MI is a kind of interpersonal communication method created by Miller and Rollnick according to the treatment experience of patients with alcohol dependence $[9,10]$. It aims to explore the contradictory psychology of patients and properly handle them. It emphasizes that the whole behavior change is a gradual process. It is believed that with the advancement of interview process, the internal motivation of patients is stronger, that is, different Therefore, different interview strategies should be adopted according to the psychological characteristics of different stages.

\subsection{Application Principles of MI}

\subsubsection{Expression of Empathy}

Also known as expression of empathy, that is, the ability of interviewers to penetrate into the heart of patients and insight into the intention of obese children is the most basic requirement of MI [5,9]. It is the basic quality of interviewers to accept and recognize the inner conflicts and conflicts of obese people through skillful feedback listening, and promote their behavior change on the basis of respecting emotion.

\subsubsection{Development Conflict}

To guide patients to find and enlarge the conflict between their pursuit of value and belief and the existing behaviors that affect their health, and spontaneously think about the possibility of achieving the goal of health through their own behavior changes. Once patients realize the contradiction between their own expectations and existing problems, they are likely to break away from the shackles of contradictions and make behavior change strategies. The focus of this process is to help children and parents establish short-term and long-term goals of weight control.

\subsubsection{Avoid Impedance}

Resistance is a normal manifestation of children's different attitudes towards behavior change. It does not mean that children are against the implementation of MI, but an expression of their own needs. To avoid conflict with children on behavior change, guide children to understand the possible results of confrontation emotion and solutions, rather than impose the subjective judgment and understanding of interviewers on children, resulting in interview deadlock.

\subsubsection{Maintaining Self-efficacy}

The individual's cognitive level of self-efficacy is an important factor in the success of behavior change [9]. By sharing weight control cases to children, they can truly realize the practical benefits brought by their own behavior mode to self growth and development changes, actively seek solutions to their own bad behaviors, and urge children to make commitment to behavior change and adhere to the behavior change plan that is ok.

\section{Application of MI in Primary Health Care of Obese Children}

\subsection{Application Population}

\subsubsection{High Baseline Weight Couples}

In addition to obese children in schools, communities and pediatric clinics, the potential population is also the focus of our attention. Parents' baseline weight, education level, eating habits and daily exercise behavior have a great impact on children's obesity, which is closely related to their health management roles in genetics, embryonic development and lifestyle introduction [11]. Parents with higher baseline weight were screened out through premarital physical examination. Interviews were conducted before marriage, during pregnancy preparation and during pregnancy, so that obese couples realized the risk of overweight on fetal growth and development, and spontaneously controlled their weight to ensure that the weight of fetus at birth was within the normal range.

\subsubsection{Life Carers of Children}

Under the traditional Chinese family model, the role of caregiver in the process of fetal growth and development 
is mainly assumed by grandparents, who dominate infant feeding and behavior. Formula feeding and overprotection make the infant weight growth rate too fast. Through MI, grandparents realize that the most dangerous problem of overweight children is the great increase in the incidence of heart disease [12], which urges them to take the initiative to think and improve the possibility of changing feeding methods to achieve the goal of health.

\subsubsection{Children with Psychosocial Impairment}

Gibson Lisa Y showed in a survey on the psychosocial level of overweight children [13]: children at the baseline level of psychosocial level have the risk of overweight and obesity in the next two years. If not timely intervention, overweight may continue to exist in the whole growth and development period, and the negative emotions brought by obesity will aggravate their psychological problems.

\subsection{Application Strategy}

The process of MI interview can be roughly divided into two stages, namely, the intention stage to enhance the intrinsic motivation of behavior change and the change stage of consolidating behavior change commitment and fulfilling behavior change plan [5]. In different stages, interview methods and techniques are also different.

\subsubsection{Intention Stage Strategy}

MI has low requirements on environmental cleanliness and the main objects are school-age children. It provides a warm, safe, fixed and interesting environment to increase the intimacy of the interviewed children, so that the interviewees can speak out their own ideas and concerns and their needs. On the principle of expressing empathy, fully understanding the children's situation and discussing the coping strategies together are the necessary conditions for intention change. For example, in the face of obese children in rural and urban-rural areas, due to the lack of access to fresh fruits and vegetables and whole grain food, sports training, limited resources to obtain professional medical care, and inconvenient transportation, etc., they are faced with practical difficulties in the intention stage [14]. Traffic subsidies, whole grain food and sports equipment are all ways to enhance intention change.

\subsubsection{Behavior Change Phase Strategy}

Strengthening the interviewee's commitment to behavior change, formulating behavior change plan and strictly implementing it are the key points of MI in behavior change stage. (1) High frequency intervention, once a week, 30 minutes for each interview, and no less than 3 months of the overall process of MI are recommended methods for weight control Mi project [15]. (2) Material incentive: organize family sports meeting, distribute prizes such as smart bracelet, weight meter and sports equipment. (3) Set up diversified training contents [16]: for example, setting up diet and exercise courses: how to distinguish between food types and calories? Introduction to a sport. (4) Guide the children and their parents to self-monitoring, record the type, amount and time of daily food intake; sports items and time; the name and quantity of sugary drinks; guide children and parents to find problems in time, formulate personalized weight loss plan, stage summary and long-term adherence.

\section{Impact Assessment}

\subsection{Evaluation of Obesity Index}

Body weight related objective indicators (BMI, waist circumference, body density, fasting blood samples, etc.) can directly and accurately reflect the effect of MI in weight control, which is less affected by subjective bias. At present, in the original research literature we refer to, in the research design, the primary outcome, namely, obesity evaluation index, has statistical significance. The disadvantage of this data design is that children's venous blood sampling has low coordination degree, high sample drop off rate [17], strong individual specificity, and the accuracy is still to be discussed. It is suggested that fasting blood sampling frequency should be 3-6 months/time or finger stick capillary blood sampling method should be used instead of venous blood sampling method to ensure sufficient sample size.

\subsection{Evaluation of Psychosocial Function}

MI can not only help children solve the problem of obesity, but also meet the emotional needs of children, making the relationship of family members more harmonious. Freiras, a 3-month randomized controlled trial of MI in adolescent weight management, showed that compared with routine weight control health education, the level of psychological and social function of children after MI intervention was significantly improved $(\mathrm{P}=0.022)$, and the level of emotional management was statistically different $(\mathrm{P}<0.001)$ [18]; similarly, Barnes A 3-month study on weight loss of primary medical institutions with MI combined with nutritional psychological education found that 
the incidence of depression and eating disorder and overeating events decreased significantly from baseline to 3 months follow-up [18]. Compared with the nutritional psychological education group, the incidence of eating disorders decreased significantly at any time after MI intervention [ $\mathrm{f}(2,53)=16.36, \mathrm{P}<0.0001]$, and depression level [t $(53)=2.79, \mathrm{P}=0.007$ [19]. There was statistical difference. It may be related to the positive emotions of the interviewees by expressing their dissatisfaction with the status quo, thinking about the existing problems and seeking solutions.

\subsection{Self efficacy Evaluation}

The application of MI enables obese children and their parents to have a correct understanding of the best possible outcome of their own efforts and external help in the process of weight control, in other words, the correct perception of self-efficacy. Bahorski JS found in a study on infant obesity risk of low-income African American mother care [20]: through Mi health education, the intervention group not only did not have excessive growth, but also did not have excessive growth. The self-efficacy of mothers of infants with obesity was higher than that of mothers of obese infants (Tukey adjusted $\mathrm{p}=0.03$ ). A nurse led Mi telephone follow-up can improve the role of parents in promoting children's health behavior The study showed that [21], compared with the conventional follow-up, MI group was able to accurately describe their goals and current progress in outpatient service at each follow-up, and their self-confidence in parents' reports was significantly increased compared with the baseline level $(\mathrm{P}=.026)$. These findings indicate that with the development of MI, the motivation of behavior change of interviewees is increasing, and the level of self-efficacy is also gradually improving, and self-monitoring is easier to be sustained.

\section{Proposal}

The positive significance of MI lies in the organic combination of psychological theory and extensive practice to prove its effectiveness in behavior change. However, the application of MI in the problem of obese children in China still needs to pay attention to the following points: (1) the selection criteria, training programs and salary of MI personnel, especially the training programs, assessment standards and the maintenance of excellent interviewers; (2) weight control To pay attention to multi-disciplinary team cooperation, we need not only clinicians and nurses, but also psychologists, educators, physiotherapists and dieticians to develop comprehensive weight control interventions according to professional knowledge; (3) systematic Mi Plan is a necessary condition to improve the quality of interviews. We can learn from some foreign research designs to establish a human body weight control for the interviewees Make files, including general information, data records of each interview and laboratory test results, long-term tracking and reducing sample loss; (4) committed to the development of weight control app, not only can reduce the workload of interviewers, but also realize the terminal real-time tracking of customer data, and regularly push weight control knowledge and interviewees' experience.

\section{Summary}

In conclusion, MI is a promising therapy in primary health care for obese children. It can not only arouse the inner motivation, change the eating habits and lifestyle, maintain the long-term motivation, but also meet the emotional needs. We should actively learn from foreign Mi models, and gradually explore interview standards suitable for China's national conditions, so as to provide practical guarantee for weight control of obese patients, and promote the further development of MI in primary health care for children with obesity.

\section{Funding}

This study was approved by Construction and Implementation of Health Management Plan for Patients with Bone and Joint Degenerative Disease (2019-PY03); Innovative Team of Integrated Traditional Chinese and Western Medicine for Prevention and Treatment of Degenerative Diseases of Bone, Joint and Spine (2019-YL02).

\section{References}

[1] Cathaoir, K. Ó. (2016). Childhood Obesity and the Right to Health. Health Hum Rights. 18(1): 249-262.

[2] Ng, M., Fleming, T., Robinson, M., et al. (2014). Global, regional, and national prevalence of overweight and obesity in children and adults during 1980-2013: a systematic analysis for the Global Burden of Disease Study 2013 [published correction appears in Lancet. 2014 Aug 30; 384(9945): 746]. Lancet. 2014, 384(9945): 766-781.

[3] Zhang, N., Ma, G. S. (2017). Interpretation of Chinese children's obesity report [J]. Journal of Nutrition, 39(06): 530-534.

[4] Zhang, N., Zhang, M., He, H. R., et al. (2019). Prevalence, influencing factors and health hazards of childhood obesity in China 
[C]. Danone Nutrition Center, 2019: childhood obesity. China Center for Disease Control and Prevention, Danone Nutrition center, 2019: 11-15.

[5] Tronieri, J. S., Wadden, T. A., Chao, A. M., et al. (2019). Primary Care Interventions for Obesity: Review of the Evidence [J]. Current Obesity Reports, 2019.

[6] Carpenter, R., Dichiacchio, T., Barker, K. (2018). Interventions for self-management of type 2 diabetes: An integrative review [J]. International Journal of Nursing Sciences, 2018.

[7] Sokalski, T., Hayden, K. A., Raffin Bouchal, S., et al. (2020). Motivational Interviewing and Self-care Practices in Adult Patients With Heart Failure: A Systematic Review and Narrative Synthesis [J]. Journal of Cardiovascular Nursing, $2020,35$.

[8] Tyler, D. O., Horner, S. D. (2016). A primary care intervention to improve weight in obese children: A feasibility study. $J$ Am Assoc Nurse Pract. 2016, 28(2): 98-106.

[9] Miller, W. R., Gribskov, C. J., Mortell, R. L. (1981). Effectiveness of a self-control manual for problem drinkers with and without therapist contact. Int J Addict. 1981; 16(7): 1247-1254.

[10] Martino, S., Ball, S. A., Nich, C., et al. (2008). Community program therapist adherence and competence in motivational enhancement therapy. Drug Alcohol Depend. 2008; 96(1-2): 37-48.

[11] Carpenter, K. M., Foote, J., Hedrick, T., et al. (2020). Building on shared experiences: The evaluation of a phone-based parent-to-parent support program for helping parents with their child's substance misuse. Addict Behav. 2020; 100: 106-103.

[12] Accurso, E. C., Norman, G. J., Crow, S. J., et al. (2014). The role of motivation in family-based guided self-help treatment for pediatric obesity. Child Obes. 2014; 10(5): 392-399.

[13] Gibson, L. Y., Allen, K. L., Davis, E., et al. (2017). The psychosocial burden of childhood overweight and obesity: evidence for persisting difficulties in boys and girls. Eur J Pediatr. 2017; 176(7): 925-933.

[14] Margaret, L., Falahee, Ramona, B., George, et al. (2016). Motivational Interviewing to Increase Physical Activity in Underserved Wome. The Journal for Nurse Practitioners. 2016, 12(10).

[15] Accurso, E. C., Norman, G. J., Crow, S. J., et al. (2014). The role of motivation in family-based guided self-help treatment for pediatric obesity. Child Obes. 2014; 10(5): 392-399.

[16] Tyler, D. O., Horner, S. D. (2016). A primary care intervention to improve weight in obese children: A feasibility study. $J$ Am Assoc Nurse Pract. 2016; 28(2): 98-106.

[17] Luque, V., Feliu, A., Escribano, J., et al. (2019). The Obemat2.0 Study: A Clinical Trial of a Motivational Intervention for Childhood Obesity Treatment. Nutrients. 2019; 11(2): 419.

[18] Freira, S., Fonseca, H., Williams, G., et al. (2019). Quality-of-life outcomes of a weight management program for adolescents based on motivational interviewing. Patient Educ Couns. 2019; 102(4): 718-725.

[19] Barnes, R. D., Ivezaj, V., Martino, S., et al. (2018). Examining motivational interviewing plus nutrition psychoeducation for weight loss in primary care. J Psychosom Res. 2018; 104: 101-107.

[20] Bahorski, J. S., Childs, G. D., Loan, L. A., et al. (2020). Parental Self-Efficacy in New Mothers Predicts Infant Growth Trajectories. West J Nurs Res. 2020; 42(4): 254-261.

[21] Schlottmann, H., Broome, M., Herbst, R., et al. (2019). Nurse-Led Telephone Follow-Up to Improve Parent Promotion of Healthy Behaviors in Young Children With Motivational Interviewing Techniques. J Pediatr Health Care. 2019; 33(5): 545-554. 